\title{
Cellular lattice Structure Produced by Selective Laser Melting and its Mechanical Properties
}

\author{
Pavel Hanzl, Miroslav Zetek, Ivana Zetková \\ Department of Machining Technolog, University of West Bohemia in Pilsen, Univerzitní 22, 30614 Pilsen, \\ Czech Republic
}

\begin{abstract}
The quest for light and stiff structures by industries such as the medical industry, the aerospace industry and other industries drives the progress in the additive manufacturing technology. This article is focused on the light-weight Schoen Gyroid cellular structure, which might offer a combination of the desired mechanical properties. The goal is to preserve the lightness of

a particular structure while maintaining the desired properties like the rigidity and solidity. Additive manufacturing has a high potential for producing these otherwise hard to make structures. The results published in this article are the obtained mechanical properties which depend on the strut thickness of the Schoen Gyroid cellular structures. These structures were additionally compared with a sample of coherent material from the same steel.
\end{abstract}

Keyword: Schoen Gyroid; Porous Structures; Rigid Construction; Additive Manufacturing; Selective Laser Melting
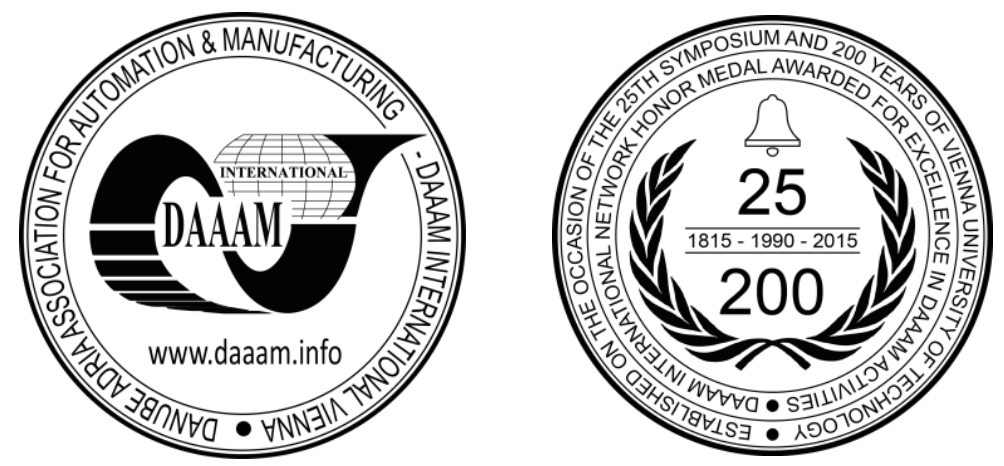

This Publication has to be referred as: Hanzl, P[avel]; Zetek, M[iroslav] \& Zetkova, I[vana] (2016). Cellular Lattice Structure Produced by Selective Laser Melting and its Mechanical Properties, Proceedings of the 26th DAAAM International Symposium, pp.0748-0752, B. Katalinic (Ed.), Published by DAAAM International, ISBN 978-3-90273407-5, ISSN 1726-9679, Vienna, Austria

DOI:10.2507/26th.daaam.proceedings. 104 


\section{Introduction}

Cellular lattice structures find wide range of usage due to their unique properties. They are used in applications where high strength accompanied by a relatively low mass is required. They also have other advantages such as thermal insulation properties and suppressed vibration. [1,2]

In recent years, certain fields of the mechanical engineering industry have been replacing solid materials with light porous materials. The interest in porous structures continues to grow. Therefore there are already several scientific articles that are involved in this area of research and development. The reference [2] was focused on brittle foam materials that were made from ceramic substrate. Used technology allows producing only irregular cellular units. These ceramic foam samples are commercially produced as ceramic filters Vukopor® which were tested.

The results of tensile strength indicated significant change in mechanical properties by comparison to the sample dimensions.

Since a regular porous structure is the main subject of this article, the additive manufacturing (AM) was chosen due to the availability in the production of highly complex metal objects. Also, because any other manufacturing approach of Schoen gyroid is not yet known. Moreover, this manufacturing technology has wide field of use. It is applied in the transition from functional prototypes to serial industrial production. This technology has even been successfully applied in repairing expensive parts. [3]

The cellular Schoen structure cannot be created by common procedures of CAD software due to the surface complexity of the gyroid. The following is an explanation. The implicit surfaces of Schoen gyroid is defined by a function. This requires implementing of other software, which is available generate surfaces described by function. A short subchapter is devoted to this topic in the article or more information is in the references $[1,4]$.

References $[1,6]$ are focused on the cellular structures of the gyroid which were produced by AM. They were interested in the manufacturability of various gyroid units in a range from $2 \mathrm{~mm}$ to $8 \mathrm{~mm}$. Their samples did not show significant defects and they demonstrated that the AM can produce very fine cellular porous structures. Although they have published valuable information on the application of AM in the gyroid production that almost did not offer any information about mechanical behaviour of gyroid structures made from maraging steel. Thus, this article adds mechanical properties of behaviour for compressive load.

When focusing on a rigid cellular structure in the context of AM, interesting outcomes may arise such as bone replacements with optimized properties or rigid structures accompanied by low weight. However, these outcomes will become applicable only after the individual mechanical behaviour of cellular structures will become known. Therefore, the aim of this article is to obtain compression mechanical properties of the Schoen Gyroid cellular structure. This experiment studies the variations in the thickness of the gyroid strut in relation to the resist of compressive loads.

\section{Study of cellular structure}

\subsection{Generation of the schoen gyroid [4]}

This phase of the research presents a method for generating a solid model of gyroid. Schoen gyroid belongs to the cubic space group I4132. It has a connectivity of three struts per unit.

The gyroid structure was described by implicit function 1.

$$
F(x, y, z)=\sin \left(\frac{2 \pi x}{a}\right) \cos \left(\frac{2 \pi y}{a}\right)+\sin \left(\frac{2 \pi y}{a}\right) \cos \left(\frac{2 \pi z}{a}\right)+\sin \left(\frac{2 \pi z}{a}\right) \cos \left(\frac{2 \pi x}{a}\right)
$$

When the $F(x, y, z)$ becomes a zero value, its points lie on the iso-surface. Variable "a" has a direct impact on the thickness of gyroid struts. Influence of the "a" variable is explained in the following paragraph.

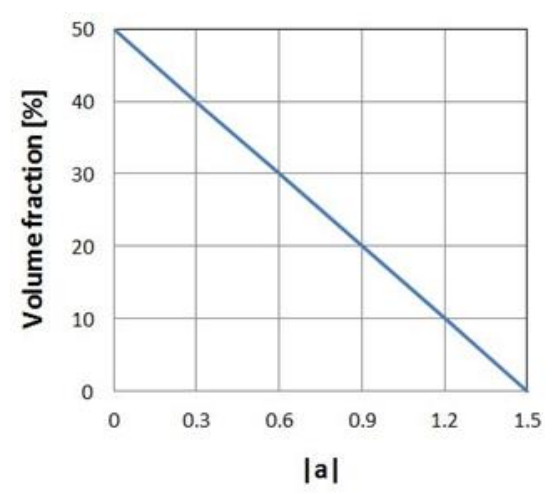

Fig. 1. Relationship between volume fraction and value of $|a|$ 
When the variable "a" is set to lower value then the struts become stronger. Conversely, when it increased, the struts became thinner. Relationship between volume fraction and value of this variable is showed in fig. 1. It shows that the value $\mathrm{a}=1.41$ is the thresh hold for manufacturability of gyroid. When this value is reached then the connection between joints of cellular lattice structure is lost.

Gyroid structures in this paper were generated as iso-surface using Matlab software (SW). By applying additional adjustments using software packages as CAD systems it achieved the creation of a solid model.

\subsection{Material}

Material used for the creation of real physical structures was defined by the European standard 1.2709. This manufactured powder was delivered by the EOS company, which calls this material EOS MaragingSteel MS1. The material composition is shown in Table 1.

\begin{tabular}{ccccccccccccc}
\hline Wt [\%] & $\mathrm{Ni}$ & $\mathrm{Co}$ & $\mathrm{Fe}$ & $\mathrm{Mo}$ & $\mathrm{Ti}$ & $\mathrm{Al}$ & $\mathrm{Cr}$ & $\mathrm{Mn}$ & $\mathrm{P}$ & $\mathrm{S}$ & $\mathrm{C}$ & $\mathrm{Si}$ \\
\hline $\mathrm{MS} 1$ & $17-19$ & $8.5-9.5$ & rest & $4.5-5.2$ & $0.6-0.8$ & $0.05-0.15$ & $<0.5$ & $<0.1$ & $<0.01$ & $<0.01$ & $<0.03$ & $<0.1$ \\
\hline
\end{tabular}

Table 1. The material composition of EOS MaragingSteel MS1 [5]

\subsection{The selective laser melting process}

The samples were produced by manufacturing systems on EOS M 290. This device uses Yb-fibre laser for melting metal powder that reaches maximum power of $400 \mathrm{~W}$. Processing conditions were predefined by EOS.

\subsection{Experiment}

This paper investigates the basic mechanical properties of Schoen gyroid. The size of unit cell was constant and was fixed at $6.5 \mathrm{~mm}$. Entire cellular lattice structures had dimensions 30x30x35 mm3. The volume fraction was set as variable. Five different lattice structures were designed with different volume fractions: $5 \%, 6.5 \%, 7.5 \%, 9.5 \%$ and $13.5 \%$.

All of the samples were successfully printed without obvious defects. Demonstration is shown in Figure 2.

The samples were subjected to compression tests on the testing device Zwick/Roell Z 250 (250 kN).

The strengths of these structures were obtained by evaluation of the tests and were entered into the Figure 3 . The individual cells usually collapsed in the $45^{\circ}$ direction $[1,1,0]$ during the test.

Computed values of the relative stiffness to the sample mass have only informative character. Aforementioned values are shown in the Figure 4, from which the following conclusion is derived. The relative stiffness to mass gradually decreases, i.e. approximately from the sample with $6.5 \%$ volume fraction of porous structure. Therefore samples with lower than $6.5 \%$ volume fraction provide inferior resistance to compressive load per unit weight.

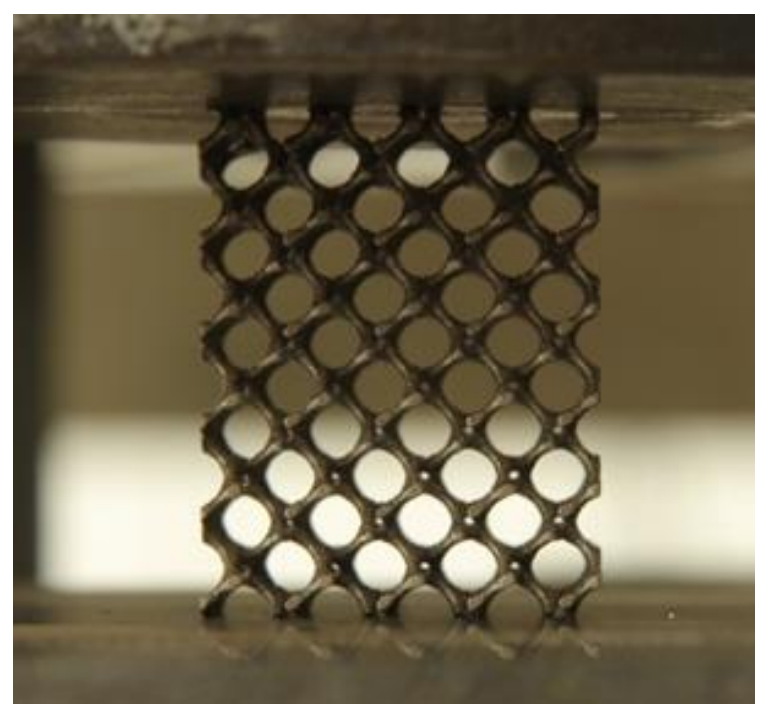

Fig. 2. The samples with $9.5 \%$ volume fraction

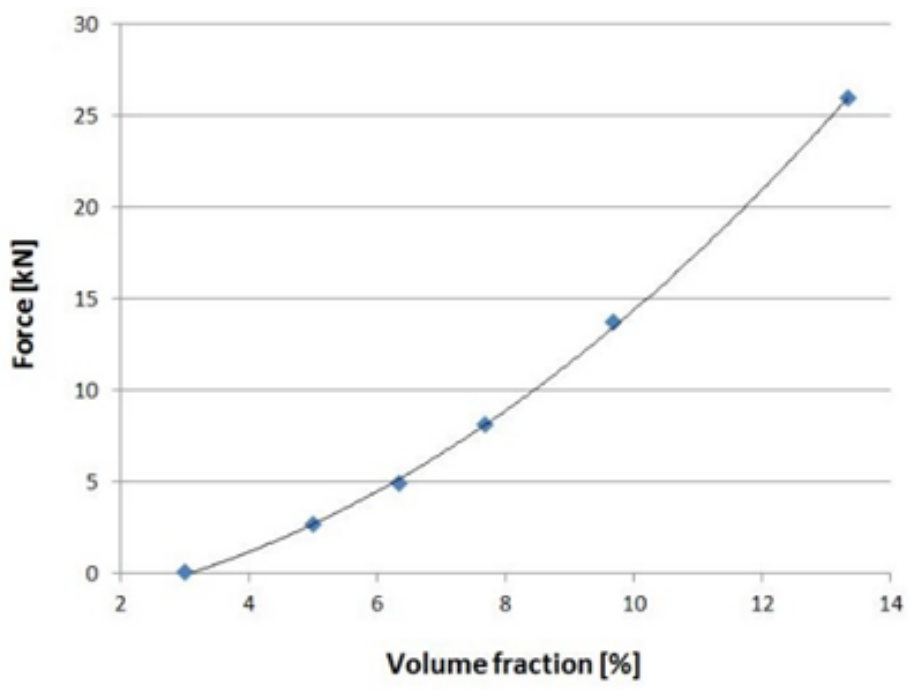

Fig. 3. The results of compressive stress 


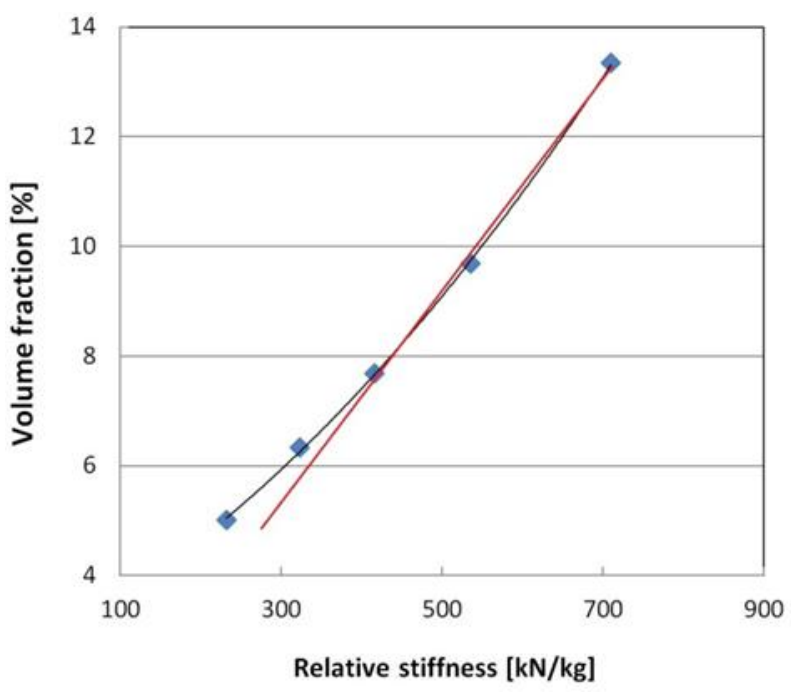

Fig. 4. The results of compressive stress.

Direct comparison of gyroid samples with the solid sample of the same dimension was not possible due to the maximum applied $250 \mathrm{kN}$ force of testing device. Assuming that the laser melted material has a tensile strength of 1000 $\mathrm{MPa} \pm 100 \mathrm{MPa}$ [5] and coefficient of 1.5 is used for conversion to a compressive strength. A theoretical force with value of $1350 \mathrm{kN}$ would be needed to cause the emergence of the plastic deformation of the same solid sample. This force corresponds to relative stiffness of $5208 \mathrm{kN} / \mathrm{kg}$ for a given weight of solid cubic.

\section{Conclusion}

The main aim of this article was to obtain mechanical properties of the cellular Schoen structure, which was the interest of several articles (see references). However, these articles almost did not offer any information about the mechanical behaviour of gyroid structures made from maraging steel, which was published by this article. All cellular lattice of Schoen gyroid were successfully produced by Selective Laser Melting. These metal samples did not exhibit any visible defects.

Investigated samples in a range of volume fractions from $13.5 \%$ to $5 \%$ showed the expected characteristics by thickness of gyroid struts. The sample with volume fraction of $13.5 \%$ had high resistance to $25.9 \mathrm{kN}$. Consequently the individual cells began to collapse in the direction $[1,1,0]$. The thinnest gyroid (5\%) from a range of tests also showed a slight resistance to $2.6 \mathrm{kN}$. The relationship between compression force and volume fraction has no linear character, but is described by a polynomial function.

The relative stiffness to weight remains constant with slight variations. However, with the exception of gyroid structures which possess smaller volume fraction of $6.5 \%$. These structures due to tapering struts showed a worse ratio of stiffness to weight. Thus, they will not probably occur in the rigid construction, but can be applied as filters with defined geometry or as a material that absorbs vibration.

Gyroid structures cannot offer comparable or similar stiffness like the solid sample with the same dimension and material. However the cellular structures of Schoen gyroid can find well-founded application in many sectors of the technical industry and can offer high rigidity accompanied by low weight. Another line of research will take including support of FEM analysis and mapping of mechanical properties depending on the direction. The result of the research will be applied in specific applications and the research will be extended by other types of cellular lattice.

\section{Acknowledgements}

This paper is based upon work sponsored by project LO1502 Development of Regional Technological Institute.

\section{References}

[1] L. Hao, D. Raymont, C. Yan, A. Hussiein, P. Young, Design and Additive Manufacturing of Cellular Lattice Structures, Innovative Developments in Virtual and Physical Prototyping - Bártolo et al. (eds), 2012 Taylor \& Francis Group, London, ISBN 978-0-415-68418-7.

[2] P. Marcian, Z. Rehorek, Z. Florian, I. Dlouhy, Estimation of the Properties Porous Structures by Experiment and Modeling, Chapter 47 in DAAAM International Scientific Book 2011, pp. 573-584, B. Katalinic (Ed.), Published by DAAAM International, ISBN 978-3-901509-84-1, ISSN 1726-9687, Vienna, Austria, DOI: 10.2507/daaam.scibook.2011.47. 
[3] T. Torims, The Application of Laser Cladding to Mechanical Component Repair, Renovation and Regeneration, Chapter 32 in DAAAM International Scientific Book 2013, pp. 587-608, B. Katalinic \& Z. Tekic (Eds.), Published by DAAAM International, ISBN 978-3-901509-94-0, ISSN 1726-9687, Vienna, Austria.

[4] S. N. Khaderi, V. S. Deshpande, N. A. Fleck, International Journal of Solids and Structures, Departmant of Engineering, Cambridge University, Cambridge, International Journal of Solids and Structures 51 (2014) 3866 3877.

[5] EOS, EOS MarigingSteel MS1, online [2015-09-27], Available from: http://www.innomia.cz/files/tinymce/files/ms1-eng.pdf

[6] Ch. Yan, L. Hao, A. Hussein, P. Young, D. Raymont, Advanced lightweight 316L stainless steel cellular lattice structure fabricated via selective laser melting, Published by Elsevier Ltd, Materials and Design 55 (2014) 533 541. 